\title{
Terapia de linguagem de irmãos com transtornos invasivos do desenvolvimento: estudo longitudinal
}

\author{
Language therapy of brothers with pervasive developmental \\ disorders: longitudinal study
}

\author{
Andréa Regina Nunes Misquiatti ${ }^{1}$, Maria Cláudia Brito²
}

\begin{abstract}
RESUMO
O objetivo deste trabalho foi descrever o processo de intervenção fonoaudiológica de dois irmãos com transtornos invasivos do desenvolvimento, por meio de um estudo longitudinal de caso clínico. Participaram dois irmãos, um de nove e outro de 11 anos de idade, ambos do gênero masculino, com autismo (Caso 1) e transtorno invasivo do desenvolvimento sem outra especificação (Caso 2). Como procedimento de coleta e análise de dados foi realizado um estudo longitudinal, por meio de acompanhamento dos casos ao longo de quatro anos de intervenção fonoaudiológica. Foram realizadas filmagens durante as sessões de terapia, análise documental de informações dos prontuários referentes à anamnese, avaliação e relatórios terapêuticos fonoaudiológicos, exames e avaliações multidisciplinares. Em ambos os casos houve melhora no contato visual, na interação social, no vocabulário e na brincadeira simbólica. No Caso 1 ocorreu aumento de 2,0 para 6,2 atos comunicativos por minuto, no Caso 2 de 3,5 para 8,0 atos e ambos demonstraram predominância do meio verbal e maior variedade de funções comunicativas. Outros fatores influenciaram estes resultados, como a deficiência intelectual, a dinâmica familiar, os conflitos no relacionamento entre os irmãos e o ambiente escolar em que estavam inseridos. Confirmou-se a relevância do fonoaudiólogo em intervenções nos transtornos invasivos do desenvolvimento, junto a equipes multidisciplinares, para a discussão diagnóstica e de condutas mais adequadas. Estudos longitudinais podem contribuir para uma análise mais detalhada e fidedigna de intervenções terapêuticas nesses casos, para esclarecer lacunas existentes na literatura e subsidiar a atuação do fonoaudiólogo clínico.
\end{abstract}

Descritores: Transtorno autístico; Reabilitação dos transtornos da fala e da linguagem; Avaliação; Relação entre irmãos

\section{INTRODUÇÃO}

O fonoaudiólogo, por tratar das alterações de linguagem, recebe diversos casos de crianças e adolescentes com transtornos invasivos do desenvolvimento (TID) que segundo a literatura ${ }^{(1)}$, se caracterizam por prejuízos severos e invasivos na interação social, na comunicação e presença de comportamentos, interesses e atividades estereotipados. Eles estão entre os transtornos de desenvolvimento mais comuns e referem-se a uma família de condições caracterizadas por grande variabilidade de manifestações clínicas que acometem mecanismos cerebrais de sociabilidade básicos e precoces ${ }^{(2)}$.

Diversos autores apontam que a terapia de linguagem

Trabalho desenvolvido no Departamento de Fonoaudiologia da Universidade Estadual Paulista - UNESP - Marília (SP), Brasil.

(1) Doutora, Professora do Departamento de Fonoaudiologia da Universidade Estadual Paulista - UNESP - Marília (SP), Brasil.

(2) Pós-graduanda (Doutorado) em Educação pela Universidade Estadual Paulista - UNESP - Marília (SP), Brasil.

Endereço para correspondência: Andréa Regina Nunes Misquiatti. Av. Hygino Muzzi Filho, 737, Caixa Postal 181, Marília (SP), Brasil, CEP: 17525-050. E-mail: amisquiatti@uol.com.br

Recebido em: 30/9/2009; Aceito em: 30/12/2009 envolvendo crianças com transtornos invasivos do desenvolvimento deve enfatizar habilidades comunicativas, interacionais e cognitivas ${ }^{(3-4)}$. Além disso, a inserção de um trabalho que envolva a famíliaa ${ }^{(5)}$, escola ${ }^{(6)}$ e uma atuação multidisciplinar colaborativa, pode contribuir de modo significativo para um melhor prognóstico em tais casos. Entre estes aspectos destacase a família como o primeiro sistema que traz implicações altamente relevantes para o desenvolvimento social, intelectual, afetivo e comportamental de seus membros ${ }^{(7)}$.

Sabe-se que a iniciação das interações de uma criança não se restringe à relação com a mãe; outros agentes sociais como irmãos, pais e avós também desempenham uma função importante no processo de desenvolvimento ${ }^{(7)}$. A interação entre irmãos pode proporcionar uma importante fonte de suporte emocional, pois o que é aprendido nesse tipo de relacionamento pode influenciar de maneira positiva ou negativa e potencializar as habilidades cognitivas, afetivas e sociais ${ }^{(8)}$.

Nesse sentido, orientações e práticas que favorecem a interação entre irmãos em programas de intervenção para crianças com transtornos invasivos do desenvolvimento podem contribuir para melhorar a qualidade de vida do paciente e sua família, especialmente quando esta é composta por mais 
de uma criança com transtorno invasivo do desenvolvimento.

Pesquisas sobre o relacionamento entre crianças com TID e seus irmãos têm enfatizado questões como qualidade de vida $^{(8)}$, estresse, depressão ${ }^{(9)}$ e habilidades sociais ${ }^{(10)}$, sendo que trabalhos com mais de uma criança com TID em uma mesma família e suas implicações no processo terapêutico ainda são escassos.

Portanto, o objetivo deste trabalho foi descrever o processo de intervenção fonoaudiológica de dois irmãos; um com diagnóstico de autismo e outro com transtorno invasivo do desenvolvimento, sem outra especificação (TID-SOE), por meio de um estudo longitudinal de caso clínico.

\section{APRESENTAÇÃO DOS CASOS CLÍNICOS}

Participaram deste estudo dois irmãos, um de nove e outro de 11 anos de idade, ambos do gênero masculino, com diagnóstico fonoaudiológico de distúrbio de linguagem, e diagnóstico psiquiátrico de autismo e transtorno invasivo do desenvolvimento sem outra especificação, respectivamente, que passaram por processo diagnóstico e terapia de linguagem em uma clínica-escola.

Como procedimento de coleta e análise de dados, foi realizado um estudo longitudinal por meio do acompanhamento dos casos, ao longo de quatro anos de intervenção fonoaudiológica. Para isso, foram realizadas filmagens durante as sessões de terapia de linguagem, além da análise documental de informações do prontuário referentes a anamnese, avaliações e relatórios terapêuticos fonoaudiológicos, exames e avaliações multidisciplinares. Vale informar que a primeira autora deste trabalho supervisionou e a segunda autora foi a terapeuta dos casos aqui descritos.

Este estudo foi autorizado mediante assinatura do Termo de Consentimento Livre e Esclarecido, elaborado para fins específicos desta pesquisa, segundo a Resolução nº. 196/96 e teve aprovação do Comitê de Ética em Pesquisa (CEP) da Universidade Estadual Paulista (UNESP) onde o trabalho foi realizado, processo n ${ }^{\circ} 368-2009$.

\section{Dados de Anamnese}

\section{Caso 1: Autismo}

A mãe procurou a clínica-escola queixando-se de atraso no desenvolvimento da fala e comportamentos "estranhos", quando a criança tinha quatro anos e cinco meses de idade. A mãe relatou intercorrências pré-natais, como episódio de hemorragia e muito "nervosismo" durante a gestação. A criança nasceu a termo, de parto normal e com icterícia, sendo necessário realizar fototerapia. Foi diagnosticado toxoplasmose na mãe e na criança, sendo que a transmissão ao paciente ocorreu durante a gestação, o que ocasionou estrabismo convergente e cicatriz macular bilateral congênita, afetando a visão central.

Segundo os relatos da mãe, seu filho apresentava sono agitado, alguns episódios de crise convulsiva e atraso no desenvolvimento neuropsicomotor. Foram observadas alterações quanto à interação social antes do primeiro ano de vida, tais como: a criança não realizava movimentos antecipatórios de levantar os braços quando queria pegá-lo; não mantinha contato visual e não demonstrava ansiedade ou medo diante de estranhos. Além disso, a mãe ressaltou que o paciente era nervoso e agitado, que não sabia brincar e batia nas outras crianças. Ela referiu que os movimentos estereotipados foram observados por volta dos quatro anos. A mãe referiu ainda atraso na aquisição da fala e linguagem; com emissão das primeiras palavras aos dois anos e seis meses de idade, bem como alterações na compreensão de ordens simples.

\section{Caso 2: Transtorno invasivo do desenvolvimento sem outra especificação}

$\mathrm{Na}$ mesma ocasião em que foi realizada a anamnese do Caso 1, a mãe também solicitou avaliação do outro filho, que tinha sete anos de idade e "falava errado". A mãe referiu que não houve intercorrências pré ou peri-natais; o paciente nasceu a termo e de parto normal. Na ocasião da anamnese a criança apresentava sono agitado, sucção digital, onicofagia e utilização de mamadeira. A mãe relatou não se lembrar das etapas do desenvolvimento motor e da aquisição de linguagem da criança. Segundo ela, seu filho começou a falar com quatro anos e na época da anamnese ainda falava "errado"; não conseguia estruturar frases, conjugar verbos adequadamente e realizava inversão pronominal. Referiu que a aprendizagem era "difícil e lenta", que brigava facilmente na escola, pois era "nervoso", "teimoso" e "medroso".

\section{Avaliações multidisciplinares}

As crianças passaram por avaliação neurológica clínica, em que foi diagnosticada deficiência intelectual em ambos os casos. Além disso, realizaram Eletroencefalograma e Tomografia Computadorizada, com resultados normais. Na avaliação otorrinolaringológica não foram observadas alterações e na avaliação audiológica por meio de métodos comportamentais, verificou-se audição normal das duas crianças. A avaliação psiquiátrica foi realizada segundo critérios específicos ${ }^{(1)}$, em que constatou-se diagnóstico de autismo no Caso 1, e no Caso 2 transtorno invasivo do desenvolvimento sem outra especificação, ambos com deficiência intelectual.

\section{Avaliação fonoaudiológica clínica}

\section{Caso 1: Autismo}

A avaliação fonoaudiológica clínica foi realizada por meio da análise de amostra de linguagem espontânea e dirigida e da observação comportamental, considerando os seguintes critérios: interação social, atividade simbólica e habilidades comunicativas. Para isso, foram oferecidos objetos lúdicos, como miniatura de animais, meios de transporte, utensílios domésticos, bola, blocos e peças para encaixar. Além disso, foi aplicado o Protocolo de Pragmática ${ }^{(11)}$, em que foram realizadas filmagens de duas sessões de 30 minutos de interação entre terapeuta e paciente, para investigar as iniciativas comunicativas, funções e meios comunicativos expressos.

$\mathrm{O}$ paciente apresentou ausência de contato visual, de simbolização e jogo social, optou por atividades isoladas e ignorou a terapeuta. Não se interessou pelos objetos oferecidos e explorou o ambiente por meio do paladar e do olfato; apresentou comportamentos repetitivos e estereotipados de 
pegar e cheirar objetos e balanceio de corpo. Verificou-se a intenção comunicativa restrita apenas com a finalidade de conseguir objetos desejados, ausência de gestos indicativos e representativos, uso de ecolalia imediata de palavras isoladas, prejuízos na compreensão de ordens simples e ausência de resposta quando chamado pelo nome. A partir da análise do Protocolo de Pragmática ${ }^{(11)}$, observou-se que a criança expressou em média 2 atos comunicativos por minuto e uso preferencial do meio gestual e das funções comunicativas Não Focalizada, Exploratória, Reativa e Pedido de objeto.

Foram observadas, portanto, alterações nas habilidades comunicativas, cognitivas e interacionais, em que se constatou distúrbio de linguagem, sendo a conduta terapia fonoaudiológica individual duas vezes por semana na própria clínica-escola.

\section{Caso 2: Transtorno invasivo do desenvolvimento sem outra especificação}

A avaliação fonoaudiológica clínica foi realizada aos sete anos, também por meio da análise da amostra de linguagem espontânea e dirigida, de observação comportamental e aplicação do Protocolo de Pragmática ${ }^{(11)}$.

A criança apresentou interação e contato visual restritos, uso convencional de objetos também explorados por meio do olfato, sem apresentar atividade simbólica. Demonstrou iniciativa e troca de turnos raros e breves, dificuldade em manter o tema do diálogo, nomeação de objetos, ecolalias, estereotipias verbais, alterações quanto à estruturação sintática, substituições e omissão de sons e prosódia inadequada. Observou-se a compreensão de ordens simples e dificuldades na interpretação de enunciados com estruturação sintática complexa. A partir da análise do Protocolo de Pragmática ${ }^{(11)}$, constatou-se que a criança expressou em média 3,5 atos comunicativos por minuto, uso preferencial do meio gestual e vocal das funções comunicativas Não Focalizada, Exploratória, Jogo, Nomeação, Pedido de objeto e Pedido de ação. Em relação à aprendizagem da leitura e escrita, a criança apresentou falta de preensão e pressão do lápis e papel, realizando apenas garatujas.

Portanto, assim como no Caso 1 verificaram-se alterações nas habilidades comunicativas, cognitivas e interacionais, sendo constatado distúrbio de linguagem, entretanto, com menor grau de severidade. Como conduta sugeriu-se terapia fonoaudiológica individual duas vezes por semana na clínica-escola.

\section{Terapia de linguagem}

O processo terapêutico de ambos os casos iniciou-se logo após a avaliação fonoaudiológica e teve duração de quatro anos. A terapia de linguagem foi fundamentada na perspectiva pragmática, que considera elementos lingüísticos e não lingüísticos para a comunicação, iniciativa de comunicação, o contexto e a participação de diferentes interlocutores. Assim, o objetivo principal foi desenvolver a competência comunicativa com ênfase no uso funcional da linguagem e na interação social.

A relação terapeuta-paciente foi fundamental para o estabelecimento de contato visual, atenção conjunta, imitação e jogo compartilhado. As situações comunicativas foram promovidas por meio de atividades lúdicas e contextualizadas, buscando explorar e atribuir funcionalidade aos objetos, desenvolver a atividade simbólica e a compreensão da linguagem oral. Foram valorizadas todas as manifestações comunicativas apresentadas, considerando também os aspectos distintos do contexto que podem influenciar o uso da linguagem. Entre tais aspectos destacam-se: o ambiente, que diz respeito ao lugar como casa ou escola; a relação entre os interlocutores, que implica as diferentes díades mãe-filho, aluno-professor e as regras interacionais envolvidas na conversação; fatores que foram investigados com a avó e a mãe das crianças.

Deste modo, o processo terapêutico procurou conduzir as crianças gradativamente aos papéis de interlocutores; favorecer a intenção comunicativa, a troca de turnos, a manutenção e a mudança de tópico conversacional, a possibilidade de informar, solicitar e narrar, buscando a maior simetria possível entre os interlocutores.

\section{Caso 1: Autismo}

A terapia de linguagem iniciou-se quando a criança apresentava quatro anos e seis meses. Durante o primeiro ano manipulava objetos de forma exploratória e solicitava a terapeuta quando desejava obter algo. Emitia de maneira contextualizada as palavras "não" e "pára"; produzia ecolalias imediatas e tardias e alguns comportamentos agressivos. Nesse período passou a utilizar funcionalmente alguns objetos e apresentou fixação por sapatos.

Foram transmitidas à criança as interpretações que a terapeuta atribuía às suas emissões orais e gestuais, enfatizando o desenvolvimento das funções comunicativas mais interativas. A terapeuta procurou sempre nomear os objetos e defini-los durante a interação, verbalizando sobre os mesmos, incentivando o paciente a manipulá-los de forma funcional, além de verbalizar as ações em que terapeuta e paciente estavam inseridos.

Nos últimos dois anos de terapia o paciente apresentou noções espaciais e de esquema corporal e imitava ações do terapeuta. Passou a chamar a terapeuta pelo nome e a elaborar frases simples contextualizadas; apresentou intenção comunicativa, iniciativa de diálogo e protonarrativa. Passou a chamar outras estagiárias e pacientes pelo nome, a interagir com a terapeuta demonstrando preferência em realizar atividade compartilhada em vários momentos; apresentou maior expressão de afetividade, aumento do contato visual e manutenção da atenção, além do uso do pronome pessoal "eu".

Outras estagiárias foram convidadas para algumas dessas atividades de interação, com o objetivo de favorecer as relações interpessoais do paciente, visto que a perspectiva funcional da linguagem considera a sociedade como elemento essencial do processo de desenvolvimento. Foram utilizados fantoches para simular conversas de diferentes pessoas dentro do contexto terapêutico. A terapeuta também procurou direcionar a atenção do paciente chamando-o pelo nome e utilizando-se de fala rica em recursos melódicos (entonação), além de procurar utilizar principalmente frases curtas e simples de modo a facilitar a compreensão do mesmo e aumentando o nível de complexidade conforme o desempenho do paciente.

Ao final do processo terapêutico, foi aplicado novamente o protocolo de pragmática ${ }^{(11)}$, sendo que a criança passou a 
utilizar 6,2 atos comunicativos por minuto, uso predominante do meio gestual e aumento dos meios vocal e verbal, passando a usar uma maior variedade de funções comunicativas como: nomeação, jogo, exclamativa, pedido de objeto, pedido de ação, pedido de rotina social, protesto, Jogo Compartilhado e Comentários.

\section{Caso 2: Transtorno invasivo do desenvolvimento sem outra especificação}

No caso do irmão mais velho com transtorno invasivo do desenvolvimento-SOE o processo terapêutico também teve duração de quatro anos, foi iniciado quando o paciente tinha sete anos, sendo concomitante ao atendimento do irmão mais jovem (Caso 1). Primeiramente foi dada ênfase aos mesmos aspectos enfocados no processo terapêutico de seu irmão, mas neste caso o direcionamento principal passou a ser o desenvolvimento de habilidades conversacionais e narrativas ${ }^{(12)}$, em função das características desse paciente.

Durante o primeiro ano de terapia as estratégias utilizadas envolveram atividades lúdicas e de interação social. Nesse período o paciente manifestou emissão de vocábulos isolados, ecolalia tardia de frases, dificuldades de compreensão oral e de interação social, uso convencional de objetos, brincadeiras com pouca criatividade, hiperatividade e déficit de atenção.

Nos dois anos seguintes as sessões passaram a envolver atividades, que visavam desenvolver a narrativa, conjuntamente ao trabalho com a atenção e a interação social. A terapeuta utilizou estratégias discursivas promovendo atividades em que se enfatizou a fala da criança a todo o momento, como miniaturas diversas, supermercado, giz de cera, pescaria, brinquedos de montar, jogos de regras, festas de datas comemorativas, corte e colagem, a fim de proporcionar um ambiente prazeroso para criança. Foram utilizados ainda livros infantis, em que a terapeuta contava as histórias ao paciente, depois solicitava que ele as recontasse, questionando "o que determinado personagem estava fazendo", "porque", "quando", "como" e "daí?". A função das perguntas é, antes de obter um relato informativo, levar a criança a organizar lembranças sob forma de discurso. A atuação da terapeuta consistiu, portanto, em dirigir, ao paciente, perguntas que, respondidas, favorecem o surgimento da narrativa.

O paciente se esquivava da terapeuta e de qualquer outra pessoa que se aproximasse, não dava as mãos e evitava ser tocado. Nessa época houve melhora na interação, a criança passou a ter iniciativa de diálogo e a realizar pequenos relatos, com alterações sintáticas e, muitas vezes, fala ininteligível, aumento das trocas de turno e diminuição das ecolalias. Com a evolução do discurso narrativo tiveram início disfluências de fala, como prolongamentos e repetições de sons, velocidade de fala aumentada, acompanhadas de tensão na região dos lábios e movimentos associados de piscar os olhos.

A partir do quarto ano de terapia as principais estratégias terapêuticas utilizadas foram conversa espontânea e diálogos sobre temas específicos durante atividades pragmáticas (construção de maquetes, livros de histórias, figuras em sequiência, filmes infantis em vídeo e diálogos sobre temas cotidianos), visto que a criança perseverava num mesmo tema. Era solicitado que o paciente relatasse fatos e elaborasse histórias. Foram introduzidas algumas sessões de terapia em grupo visando complementar o trabalho com a interação social.

Além disso, as habilidades sociais foram trabalhadas no sentido de melhorar a percepção do contexto situacional e da perspectiva do ouvinte. De tal modo, foram realizadas estratégias com o objetivo de propiciar que a criança percebesse os efeitos que seu comportamento e discurso poderiam causar no interlocutor. Assim a criança foi exposta a dramatizações de situações cotidianas e histórias infantis, com melhora nas habilidades conversacionais e de narrativas.

No decorrer do processo terapêutico foi observado que o relacionamento entre os irmãos influenciava significativamente o comportamento de ambos, tanto em âmbito terapêutico, como em outros ambientes, segundo relatos da mãe. Notouse que o irmão mais velho demonstrava mais agressividade, agitação e aumento de comportamentos repetitivos, pelo fato da mãe evidenciar maiores cuidados e atenção em relação ao filho mais novo. $\mathrm{O}$ filho mais novo reagia à agressividade do irmão apresentando-se mais ansioso e agitado.

Ao término do processo terapêutico, novamente aplicou-se o Protocolo de Pragmática ${ }^{(11)}$, obtendo o resultado de 8,0 atos comunicativos por minuto, uso predominante do meio verbal e das funções comunicativas, pedido de informação, pedido de ação, pedido de rotina social, comentários e narrativa.

\section{Orientação e alta fonoaudiológica}

Foram realizadas discussões com psicólogos e orientações sistemáticas à família, inclusive para favorecer a interação entre os irmãos, sendo também questionado regularmente a respeito das possíveis mudanças que ocorreram com as crianças em ambiente familiar e escolar. A mãe foi esporadicamente convidada a entrar na sala de terapia e participar de algumas situações de interação.

A orientação fonoaudiológica incluiu ainda assessoria à escola, para que este local também fosse um ambiente favorável ao desenvolvimento de suas habilidades comunicativas e sociais, sendo imprescindível uma atuação interdisciplinar e colaborativa.

Quanto à alta fonoaudiológica, ressalta-se que esse procedimento pode variar de acordo com as particularidades de cada caso, como o nível de desenvolvimento do paciente, o envolvimento familiar, o tipo de processo educacional no qual está inserido, atendimentos multidisciplinares, contexto sócio-econômico, entre outros aspectos que podem ser levados em consideração.

$\mathrm{Na}$ época em que se completaram quatro anos de intervenção fonoaudiológica, a escola regular sugeriu que as crianças realizassem avaliação em uma instituição de educação especial. Tal instituição oferecia atendimento clínico e educacional, assim discutiu-se a possibilidade da alta fonoaudiológica assistida da clínica-escola e a transferência das crianças para essa instituição.

Em ambos os casos a alta assistida foi realizada, com acompanhamento por meio de retornos inicialmente a cada três meses, depois a cada seis meses e então anuais. Nos retornos foram realizadas avaliações, para observar novas aquisições e novas habilidades que podem ser desenvolvidas e com base nisso foram oferecidas orientações específicas aos familiares. 


\section{DISCUSSÃO}

Os achados deste estudo confirmam dados da literatura referentes ao autismo e ao TID-SOE, que podem ou não estar associados à deficiência intelectual ${ }^{(2)}$, sendo que os casos aqui descritos apresentaram esta comorbidade, o que influenciou na utilização de determinadas estratégias e em seus prognós$\operatorname{ticos}^{(13)}$.

A intervenção adotada neste estudo foi individualizada e conduzida de acordo com as habilidades e dificuldades de cada criança, o que também é apontado por outros autores ${ }^{(14)}$. Foi possível verificar ainda a evolução do desenvolvimento de linguagem, cognitivo e social dos dois irmãos por meio de uma prática de intervenção, que priorizou aspectos interacionais e funcionais da linguagem, baseada em uma perspectiva pragmática $^{(14)}$.

Em relação aos resultados terapêuticos de cada caso, o atendimento de ambos, ocorreu a partir da construção do vínculo entre terapeuta e paciente ${ }^{(15)}$. A busca por uma relação o mais simétrica possível entre a criança e o terapeuta para a construção da linguagem, a criação de situações de atenção conjunta e de jogo compartilhado $^{(4)}$ foram fundamentais nesse processo, além de favorecer o desenvolvimento de habilidades cognitivas.

No Caso 1, da criança com autismo, primeiramente a ênfase ocorreu na comunicação não-verbal (expressões faciais, utilização de gestos) e em atividades lúdicas que buscavam favorecer a inserção da criança em diferentes contextos sociais, conforme indicado por outros autores ${ }^{(3-4)}$. No decorrer do processo e com o desenvolvimento da linguagem verbal da criança, foram inseridas estratégias que envolveram iniciativa e manutenção do diálogo, troca de turnos e desenvolvimento da narrativa ${ }^{(12)}$.

No que se refere ao Caso 2, da criança com transtorno invasivo do desenvolvimento sem outra especificação, no início foi dada ênfase nos mesmos aspectos enfocados no processo terapêutico de seu irmão, mas neste caso o direcionamento principal passou a ser o desenvolvimento de habilidades conversacionais e narrativas ${ }^{(12)}$, em função das características desse paciente.

Ao final do processo terapêutico verificou-se que no Caso 1 houve aumento de 2,0 para 6,2 atos comunicativos por minuto, enquanto que no Caso 2 ocorreu aumento de 3,5 para 8,0 atos comunicativos por minuto e ambas as crianças demonstraram predominância no uso do meio verbal e maior variedade de funções comunicativas. Estes achados foram também observados em estudos anteriores, que verificaram melhora nessas três categorias de análise (atos, meios e funções comunicativas) ao investigar o perfil comunicativo nos TID após período de terapia fonoaudiológica ${ }^{(14)}$.

Sugere-se também a possibilidade de outros fatores terem influenciado o desenvolvimento da comunicação das crianças, como a deficiência intelectual ${ }^{(13)}$, a dinâmica e o envolvimento familiar $^{(5,14)}$, os conflitos no relacionamento entre os irmãos ${ }^{(8)}$ e o ambiente escolar ${ }^{(6,14)}$ em que estão inseridos.

Quanto ao procedimento de alta fonoaudiológica assistida, tema ainda pouco discutido na literatura nacional, a conduta foi direcionada não apenas com base na evolução dos casos; mas foram priorizadas as necessidades desta família, relacionadas à inserção dos dois irmãos em uma instituição de educação especial e a sobrecarga materna no que se refere aos cuidados com os filhos. Como afirma a literatura em processos terapêuticos de longa duração, no caso de indivíduos autistas é necessário considerar os resultados obtidos a partir de interferências pontuais ${ }^{(14)}$.

\section{COMENTÁRIOS FINAIS}

Neste estudo foi possível confirmar que a opção pela abordagem pragmática para fundamentar a terapia de linguagem mostrou-se eficaz, pois permitiu considerar as particularidades de comunicação e interação dos casos aqui descritos. Além disso, notou-se que orientações claras e objetivas à escola e à família acerca desses aspectos e do relacionamento entre os dois irmãos foram fundamentais.

Foi possível ainda apresentar neste trabalho o procedimento de alta fonoaudiológica assistida, o que possibilitou suporte adicional à família e à instituição educacional na qual as crianças com TID estavam inseridas, tema que ainda é pouco discutido na literatura nacional e que ainda suscita muitas dúvidas entre estudantes e profissionais.

Vale destacar ainda a importância de se documentar dados de terapia fonoaudiológica não apenas por meio de relatos escritos, mas também por registros audiovisuais, que contribuem para uma análise mais detalhada e fidedigna da evolução dos casos e para o desenvolvimento de estudos longitudinais, que permitem esclarecer lacunas existentes na literatura e subsidiar a atuação do fonoaudiólogo clínico. 


\begin{abstract}
The aim of this research was to describe the language intervention process of two brothers with pervasive developmental disorders, through a longitudinal clinical case study. Two brothers - one nine and the other 11 years old - participated in the study. Subjects had diagnosis of autism (Case 1) and pervasive developmental disorder-not otherwise specified (Case 2), respectively. As a procedure for data collection and analysis, a longitudinal study was carried out through a follow up of the cases during the four years of language therapy. The procedure included filmed therapy sessions, documental analysis of information contained in the patients' records regarding anamnesis, assessments and therapeutic reports, and multidisciplinary evaluations and exams. In both cases there was an improvement on visual contact, social interaction, vocabulary and symbolic play. For Case 1, there was an increase from 2.0 to 6.2 communicative acts per minute; for Case 2, an increase from 3.5 to 8.0 acts. Both subjects demonstrated predominance of the verbal communicative mean and greater variety of communicative functions. Other factors influenced these results, such as the intellectual deficiency, the family dynamics, the conflicts on the relationship between the brothers and the school environment in which they were placed. The study confirmed the relevance of the speech-language pathologist in interventions of pervasive developmental disorders, along with multidisciplinary teams, for the discussion of diagnoses and of the most appropriate procedures. Longitudinal studies can contribute for more detailed and reliable analysis of therapeutic intervention in these cases, in order to clarify gaps existent in literature and to support the role of clinical speech-language pathologists.
\end{abstract}

Keywords: Autistic disorder; Rehabilitation of speech and language disorders; Evaluation; Sibling relations

\section{REFERÊNCIAS}

1. American Psychiatric Association - APA. Manual diagnóstico e estatístico de transtornos mentais - DSM-IV-TR. 4a ed. Porto Alegre: Artmed; 2002.

2. Klin A, Mercadante MT. Autismo e transtornos invasivos do desenvolvimento. Rev Bras Psiquiatr. 2006;28(Supl.1):s1-s2.

3. Jones LA, Campbell JM. Clinical characteristics associated with language regression for children with autism spectrum disorders. J Autism Dev Disord. 2010;40(1):54-62.

4. Volden J, Coolican J, Garon N, White J, Bryson S. Brief report: pragmatic language in autism spectrum disorder: relationships to measures of ability and disability. J Autism Dev Disord. 2009;39(2):38893.

5. Tager-Flusberg H, Rogers S, Cooper J, Landa R, Lord C, Paul R, et al. Defining spoken language benchmarks and selecting measures of expressive language development for young children with autism spectrum disorders. J Speech Lang Hear Res. 2009;52(3):643-52.

6. Woodcock A, Woolner A, Benedyk R. Work. Applying the HexagonSpindle Model to the design of school environments for children with Autistic spectrum disorders. Work. 2009;32(3):249-59.

7. Dessen MA. A família como contexto de desenvolvimento. In: Fleith DS, organizador. A construção de práticas educacionais para alunos com altas habilidades/superdotação. Vol. 3, O aluno e a família. Brasília: Ministério da Educação, Secretaria de Educação Especial. 2007; p. 1327.

8. Marciano AR. Qualidade de vida de irmãos de portadores de autismo infantil. In: Autismo infantil: novas tendências e perspectivas. Assumpção Júnior FB, Kuczynski E. São Paulo: Atheneu; 2007. p. 25565 .
9. Gomes FV, Bosa C. Estresse e relações familiares na perspectiva de irmãos de indivíduos com transtornos globais do desenvolvimento. Estud Psicol (Natal). 2004;9(3):553-61.

10. Toth K, Dawson G, Meltzoff AN, Greenson J, Fein D. Early social, imitation, play, and language abilities of young non-autistic siblings of children with autism. J Autism Dev Disord. 2007;37(1):145-57.

11. Fernandes FD. Pragmática. In: Andrade CR, Befi-Lopes DM, Fernandes FD, Wertznner HF. ABFW: teste de linguagem infantil nas áreas de fonologia, vocabulário, fluência e pragmática. Carapicuiba: Pró-Fono; 2004; p. 83-97.

12. Fernandes FD. Os atrasos na aquisição de linguagem. In: Goldfeld M, organizador. Fundamentos em fonoaudiologia: linguagem. 2a ed. Rio de Janeiro: Guanabara Koogan; 2003. p. 23-38.

13. Lidstone JSM, Fernyhough C, Meins E, Whitehouse AJ. Brief report: Inner speech impairment in children with autism is associated with greater nonverbal than verbal skills. J Autism Dev Disord. 2009;39(8):1222-5.

14. Fernandes FD, Cardoso C, Sassi FC, Amato CH, Sousa-Morato PF. Fonoaudiologia e autismo: resultado de três diferentes modelos de terapia de linguagem. Pró-Fono. 2008;20(4):267-72.

15. Marques CF, Arruda SL. Autismo infantil e vínculo terapêutico. Estud Psicol. (Campinas). 2007;24(1):115-24. 